\title{
Analyzing and Comparing the Effect of Water and Surfactant Cleaning on Performance of Solar Photovoltaic Panels
}

\author{
Tripti*, Ankit Agarwal, Chandan Kumar \\ Department of Mechanical Engineering, Swami Keshvanand Institute of Technology, Management \& \\ Gramothan, Jaipur-302017 (INDIA) \\ Email: tkumawat.2april@gmail.com*, akagarwal.87@gmail.com, chandanpink1988@gmail.com \\ Received 15.01.2021, received in revised form 23.01.2021, accepted 28.01.2021
}

\begin{abstract}
In the present study the surfactant based cleaning and water based cleaning systems have been fabricated and both systems were compared with system without washing. The experimental investigations are done in the month of March, April and May (from 4th March to 1st June) to analyze the performance enhancement. The surfactant and water based system contains manual cleaning by mixing surfactant in water. Power and efficiency were compared.
\end{abstract}

Keywords- Cleaning, Power Generation, Solar Panel

\section{INTRODUCTION}

As per the Renewable Energy Attractiveness Index 2018 the renewable energy sector in India is the fourth most attractive market of renewable energy in the world. India looks to meet 15,820 TWh energy demand on its own by 2040. By 2030, $40 \%$ of power demand will be accomplished by renewable energy sources [1]. The country's solar installed capacity was 253.94 MW on roof top and 1114.75 MW on ground mounted as of 30 June 2019. India has become globally the lowest cost producer of solar power [2].

\subsection{Factors affecting energy conversion efficiency of $P V$ panels}

All of the sunlight that reaches on PV cell is not converted into electricity. Hence, the efficiency of PV panels is very low. To enhance the efficiency of PV panels effect of various factors that reduces the exposure of sunlight to PV panels are considered [3]. Wrong orientation could affect efficiency of solar power. If solar panels were not positioned in right direction, rays hitting the panels will decreases that will definitely decreases its efficiency. For getting maximum efficiency, solar panels were mounted at an angle equal to latitude of the place of installation so that sunrays will hit normal to the surface of the panel.

Accumulation of dust on the solar panels also decreases the efficiency of panels. As the dust on panels increases day by day the accumulated dust on the solar panel blocks the sun's rays which results in decrement of efficiency of solar panel that results in low performance of the solar panel until the panels are cleaned manually or by rain. So cleaning of solar panels regularly to maintain efficiency plays a vital role.

A very high temperature can also lead to decrease in the output of solar panel thus can decreases its efficiency As the temperature rises, current in solar panel increases but voltage of solar cell decreases. The decrease in voltage is more than that of increase in current so overall power output is reduced which results in decrease in efficiency. Efficiency also depends upon crystal structure of solar panel. Solar cells made from a single crystal (Monocrystalline) of silicon makes them highly efficient while solar panels have solar cells made from many fragmented silicon (polycrystalline) melted together making their efficiency lesser than monocrystalline.

\subsection{Methods of cleaning of solar panel}

Solar panels can be cleaned by a forced airflow. The forced air removes the particles of dust from the surface of PV panels but this also requires more energy to produce pressurized air. Fig.1 shows a conceptual diagram of this type of system.

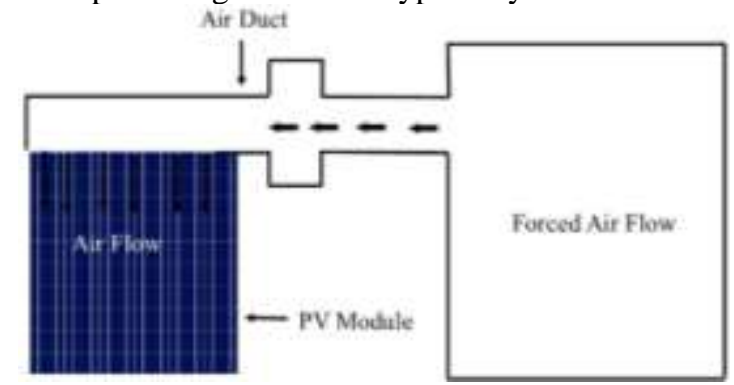

Fig.1 Conceptual diagram of forced air flow based cleaning

Pressurized water can also clean solar panels. In this method solar panels are cleaned by pressurized water. To pressurize the water lot of power is required along with large quantity of water requirement to clean panels; these two factors are main drawback of this method. Also the solar panel attracts more dust when they are wet after cleaning. Mechanical instruments can also be used for cleaning purpose. In this method mechanical instrument like a robotic brush is used to clean the panel. Cleaning can be done with or without water with help of brushes. Patil P.A. et al. [4] 
mathematically optimized the dust cleaning equipment. The optimization is done on cleaning brush design. The experiment concluded that optimized brush increased the efficiency of panel from 9 to $11.6 \%$. Mechanical cleaning reduces the labor cost as it uses the automatic system which is composed of sensors, micro controllers etc. The efficiency of this method is not high in areas where dust accumulation is high. The drawbacks of this method can be reduced by using motors which requires less power to operate brushes.

Solar panels can also be cleaned by Electrodynamic screen (EDS).This method doesn't require any mechanical instrument or water for cleaning purpose. Kawamoto H. [5] experimentally studied the Electrostatic cleaning by equipment. In this method a high voltage is applied between the ends of solar panel. The high voltage generates the electric field which produces small vibrations in the dust particles and thus the dust is removed by shifting to the edge of panel. EDS screen cleaning is shown in fig 2 .

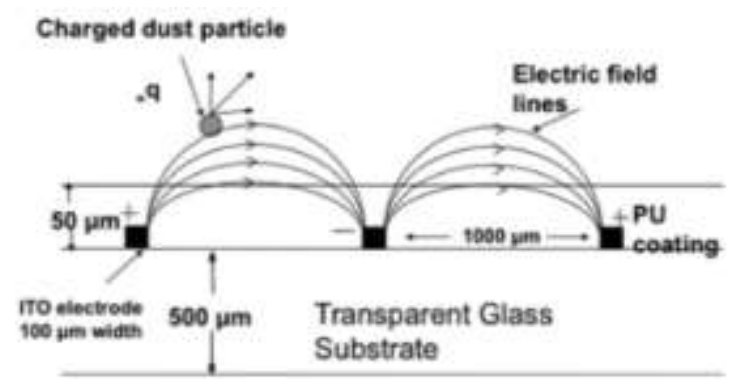

Fig.2 EDS screen cleaning

Kawamoto H. and Guo B. [6] also proposed improved cleaning system for dust on solar panel. The experiment used Namib sand and Doha dust. The high voltage is generated by positive and negative amplifiers switches and applied between the glass substrate and glass cover. The set up concluded that high voltage improves the cleaning performance. Solar panel insulation must be improved to handle high voltage.

Self-cleaning coatings also plays important role in solar panel cleaning. In this method nano-patterned fabrication and chemical coating is done on glass surface. Mozumder M.S. et al. [7] reviewed the selfcleaning coatings for solar panel cleaning. Superhydrohphobic and superhydrophillic coatings were studied in detail. Solvent based method which includes Sol-gel method and Dip coating, Electro spinning Vapor-assisted coating method which includes Plasma etching and Chemical vapor deposition and Dry powder coatings were studied to apply coatings on solar panel. In this method hydrophobic or hydrophilic surface is created by chemical coating/screen layer. The coating doesn't allow dust to stick on the surface of panels and chemically breakdown the organic dirt by reacting with UV rays. Walz K.A. et al. [8] experimentally studied the effect of self-cleaning coating which comprises of titanium oxide ( $\mathrm{TiO} 2)$ and silicon oxide ( $\mathrm{SiO} 2)$ with solution gel chemical processing. The study shows that self-cleaning coatings can be successfully applied on field for cleaning purpose. The experiment didn't concluded any result but showed that self-cleaning coating can be studied more to improve the efficiency of solar panel. Degradation of coating is the main drawback of selfcleaning coatings.

In surfactant cleaning method the surface is cleaned by using water mixed with a surfactant. Surfactant is a wetting agent that lowers the surface tension of water and allows water easier spreading. This is an improvised version of water based cleaning system. As the dust particles contain positive, negative and neutral particles surfactants are capable of removing these charged and uncharged particles as well.

Rain fall cleaning is a natural process of cleaning. The rainfall cleaning leaves smaller dust particles on the panels. These smaller dust particles can be removed heavier rainfall. For getting consistent efficiency in power generation the requirement is complete and timely cleaning of solar panels.

In this study, solar PV panels were cleaned by using water and a surfactant which is implemented practically on installed PV panels in Jaipur, Rajasthan, India. To test the applicability of the experiment in water abundant areas and dusty areas like Rajasthan the experiment used non pressurized water. The next section describes the experimental setup and the steps required to perform the experiment.

\section{EXPERIMENT SETUP}

A experiment set up is made by using photovoltaic (PV) panels to study the solar cleaning methods i.e cleaning using water only, cleaning using water mixed with surfactant and no cleaning. Fig. 3 shows the experimental set up.

The characteristics of the PV panels are shown in Table 1.

The experiment includes three main modules which are as follows:

1. Three solar PV Panels of $20 \mathrm{~W}$ peak output each.

2. 5L capacity water inlet and PVC pipes for cleaning.

3. Multimeter for measuring current and voltageZhao et. al. surveyed

\section{METHOD OF EXPERIMENT}

Three panels were taken for performing experiment. Panel A is for without washing system, Panel B is for washing by water and panel $\mathrm{C}$ is for washing surfactant (Zwitterionic) [9] mixed in water. 
These panels are mounted on the roof top at $26^{\circ}$ angle to the horizontal surface facing towards south. Two water tanks and PVC connections are used for cleaning of panels B and C. One water tank is filled with water to clean panel B and another water tank is filled with mixture of water and surfactant with concentration of $1 \mathrm{gm}$ in 2 liter to clean panel $\mathrm{C}$.

A multimeter is used to measure voltage (Voc) and current (Isc) after an interval of every 1 hour from 9 am to 5 pm daily.

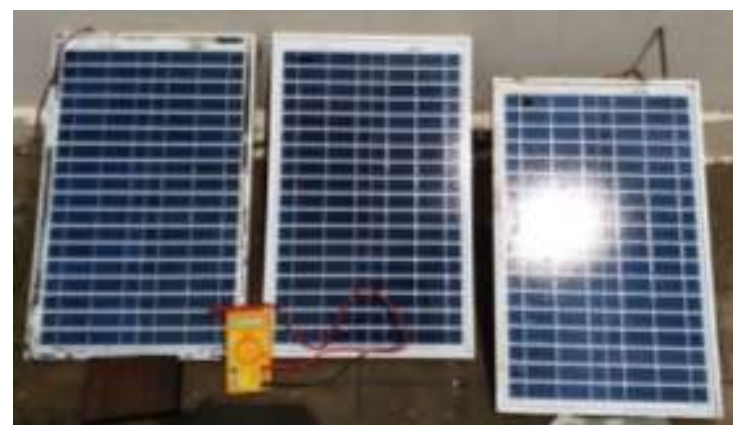

Fig.3 Pictorial view of experimental setup

Table 1 Characteristics of the PV modules

\begin{tabular}{|l|l|}
\hline Characteristics & Value/Tested \\
\hline Peak Power $(0-4.99 \mathrm{Wp})$ Pmax $(\mathrm{Wp})$ & 20 \\
\hline Open Circuit Voltage $\mathrm{V}_{\mathrm{oc}}(\mathrm{V})$ & $21.44 / 22.18$ \\
\hline Short Circuit Current $\mathrm{I}_{\mathrm{sc}}(\mathrm{A})$ & $1.27 / 1.46$ \\
\hline Maximum Voltage $\mathrm{V}_{\mathrm{mpp}}(\mathrm{V})$ & $17.15 / 18.03$ \\
\hline Maximum Current $\mathrm{I}_{\mathrm{mpp}}(\mathrm{A})$ & $1.18 / 1.36$ \\
\hline Module Efficiency $(\%)$ & $10.23 / 12.54$ \\
\hline $\begin{array}{l}\text { Temperature coefficients }\left(\mathrm{T}_{\mathrm{c}}\right) \text { of } \\
\text { Open Circuit Voltage }(\beta)\end{array}$ & $-0.31 \% /{ }^{\circ} \mathrm{C}$ \\
\hline $\begin{array}{l}\text { Temperature coefficients }\left(\mathrm{T}_{\mathrm{c}}\right) \text { of } \\
\text { Short Circuit Current }(\alpha)\end{array}$ & $0.058 \% /{ }^{\circ} \mathrm{C}$ \\
\hline Fill Factor & $0.74 / 0.76$ \\
\hline Length $\times$ Width $\times$ Height & $\begin{array}{l}552 \mathrm{~mm} \times 354 \text { mm } \times 18 \\
\text { mm }\end{array}$ \\
\hline Weight & $2.8 \mathrm{Kg}$ \\
\hline Cells & $\begin{array}{l}36 \text { Polycrystalline solar } \\
\text { cells, } 2 \mathrm{BB} / 3 \mathrm{BB}\end{array}$ \\
\hline Frame & $\begin{array}{l}\text { Anodized aluminium } \\
\text { frame with twin wall } \\
\text { profile }\end{array}$ \\
\hline Application Class & Class A $($ Safety class II) \\
\hline
\end{tabular}

The data of solar radiation intensity $(\mathrm{G})$ is taken from MNIT whether data [4] on which the data are available at interval of every 15 minutes daily.

The efficiency is the calculated by formula given in equation 1

$$
\begin{aligned}
& \text { Efficiency } \\
& =\frac{\text { Daily average power generated }}{\text { Average daily solar radiation } \times \text { Area of panel }}
\end{aligned}
$$

The panels were cleaned at interval of every 7 days. Table 2 represents the dates on which cleaning is done manually and dates on which the rainfall occurred.

The total power generated during the experiment i.e. from $4^{\text {th }}$ March to $1^{\text {st }}$ June 2019 for all three panels were calculated by formula give below:

Power $=$ Voc $\times$ Isc $\times$ Fill factor
Table 2 Dates of cleaning panels

\begin{tabular}{|c|c|}
\hline Washing count & Dates of cleaning \\
\hline Start of experiment & $4-3-2019$ \\
\hline 1 & $7-3-2019$ \\
\hline 2 & $14-3-2019$ \\
\hline 3 & $21-3-2019$ \\
\hline 4 & $28-3-2019$ \\
\hline 5 & $4-4-2019$ \\
\hline 6 & $11-4-2019$ \\
\hline 7 & $18-4-2019$ \\
\hline 8 & $25-4-2019$ \\
\hline 9 & $2-5-2019$ \\
\hline Rainfall & $3-5-2019$ \\
\hline 10 & $9-5-2019$ \\
\hline 11 & $16-5-2019$ \\
\hline 12 & $23-5-2019$ \\
\hline 13 & $30-5-2019$ \\
\hline End of experiment & $1-6-2019$ \\
\hline
\end{tabular}

\section{PROPOSED WORK}

\subsection{Efficiency}

The average daily efficiency of the system A, B and $\mathrm{C}$ is shown in fig. 4. From figure, it was observed that system $\mathrm{C}$ maintained its efficiency consistently while system A and system B, efficiency decreases due to dust deposition. The overall efficiency of System A was decreased by $29 \%$ and the efficiency of system B and system C was decreased by $18.17 \%$ and $5.2 \%$ respectively. The efficiency of system $\mathrm{B}$ is decreased with a slope of 0.03/day. As the system A is not washed the efficiency of system $\mathrm{A}$ is decreasing significantly with a slope of $0.07 /$ day. Natural rain occurred at 3rd May a spike in efficiency of system A is clearly observed because system A is not washed manually. Rain water cleaned the surface of system A only up to certain extent.

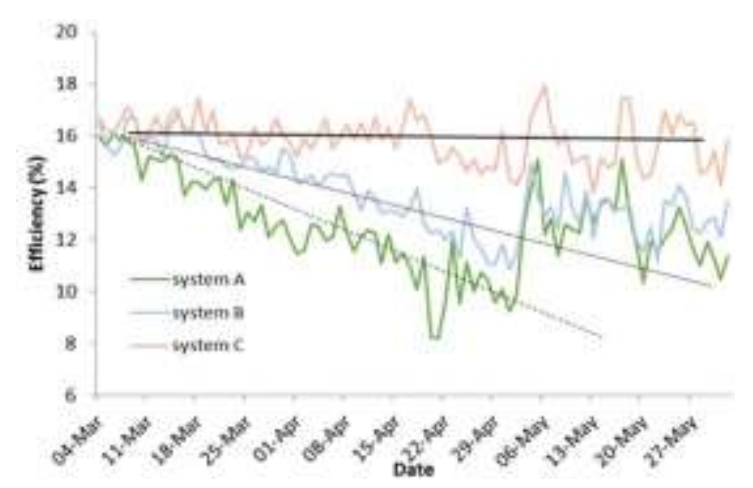

Fig.4 Average daily efficiency of the system A, B and C

\subsection{Total maximum power generation}

The total maximum power generation (in $\mathrm{kWh}$ ) by all three systems during the experiment is shown in fig.5. From figure; it was observed that the power generated by system $\mathrm{A}$ is $10.81 \mathrm{kWh}$ in 90 days (March 04 to June 01, 2019). The system B generated $11.95 \mathrm{kWh}$ and system $\mathrm{C}$ generated 13.78 $\mathrm{kWh}$. This means the system $\mathrm{C}$ generated $27.5 \%$ 
more power than system $\mathrm{A}$ and $15.3 \%$ more power than system B in 90 days.

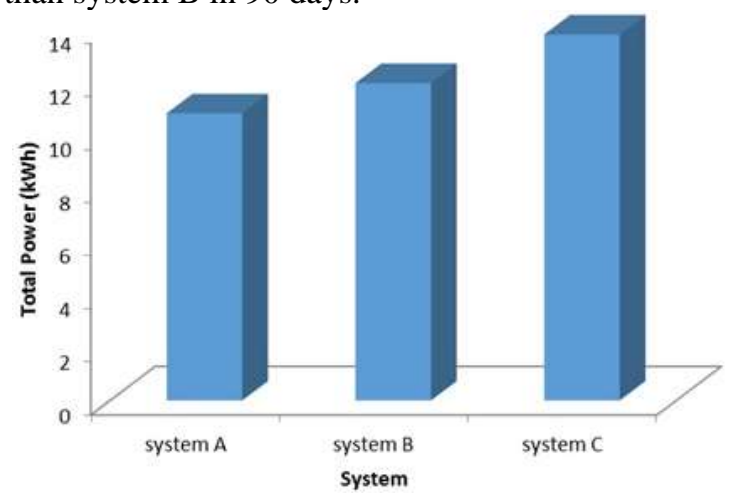

Fig.5 Total power generation (in $\mathrm{kWh}$ ) by systems A, B \& C during the experiment

\subsection{Dust deposition on surface of panels}

The airborne dust is deposited continuously on the PV panels. As the dust deposited, it reduces the performances of PV panels. fig.6, 7 and 8 shows the surface of panels after 7 days, 30 days and 60 days respectively.

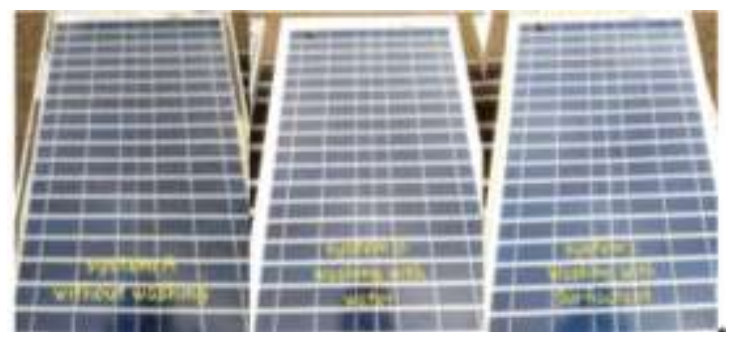

Fig.6 Dust deposition after 7 days

After 7 days of operation a little dust is deposited on panel A while a little to no dust is deposited on panel $\mathrm{B}$ and on panel $\mathrm{C}$ there was no dust deposition. After 30 days we found a thick dust layer on panel A while on panel B a very thin layer of dust is observed and panel $\mathrm{C}$ was clean and no dust particles were observed on surface of panel C. After 60 days of operation a very thick layer of dust was observed on panel $\mathrm{C}$ while on Panel B a significant dust layer of very small dust particles with stains of water were observed and panel $\mathrm{C}$ was still clean.

\subsection{Economic Survey}

The cost for surfactants used for cleaning of system $\mathrm{C}$ can be found as follows:

Cost of surfactant / kg (INR) $=98$

Total surfactant used in single wash $=1 \mathrm{gm}$

Total wash count in 90 days of experiment $=13$

Total cost of surfactants during experiments $=$ $98 * 0.001 * 13=1.274 /-$

Thus by spending INR 1.274 , we are able to generate $27.5 \%$ more power in 90 days.

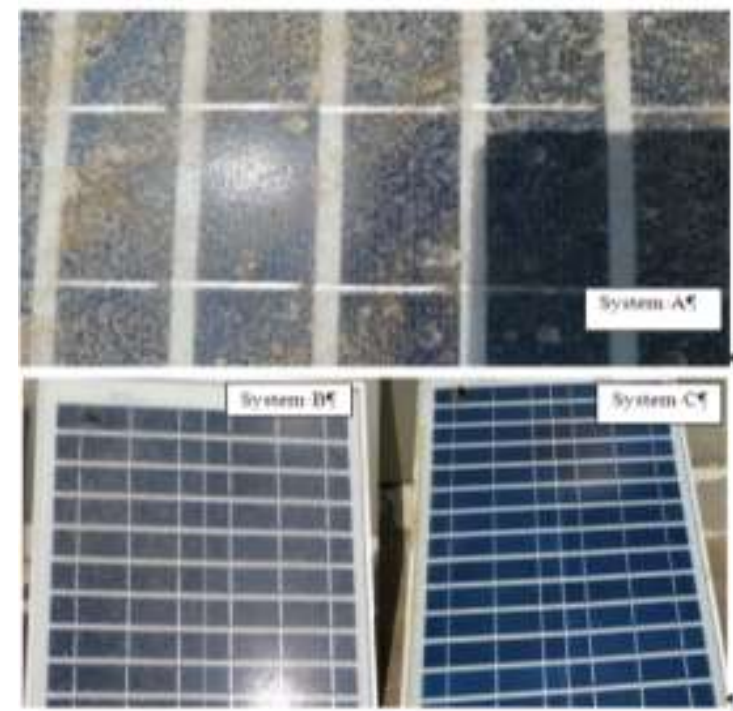

Fig 7 Dust deposition after 30 days
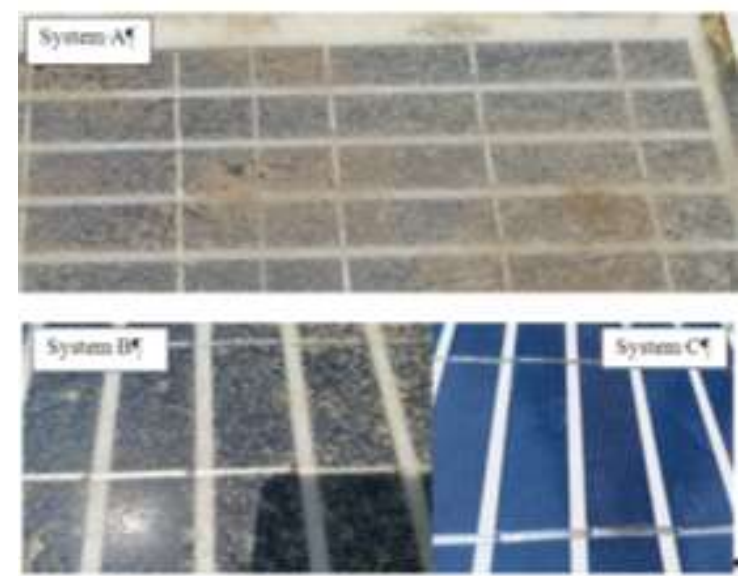

Fig.8 Dust deposition after 60 days

\section{CONCLUSION}

The total maximum power generation by system $\mathrm{C}$ washed by surfactant mixed with water was 13.78 $\mathrm{kWh}$, and by system B and system A, it was 11.95 $\mathrm{kWh}$ and $10.81 \mathrm{kWh}$ respectively. This means the system $\mathrm{C}$ generated $27.5 \%$ more power than system A and $15.3 \%$ more power than system B in 90 days. The overall efficiency of system $\mathrm{C}$ washed with surfactant was higher than that of system B washed with water. The overall efficiency of System A was decreased by $29 \%$ and the efficiency of system B and system $\mathrm{C}$ was decreased by $18.17 \%$ and $5.2 \%$ respectively. Surfactant washing gives the consistent efficiency.

Dust deposition was found minimum on system $\mathrm{C}$ as the surfactant mixed with water removes all dust from panel. On system B, a thin dust layers was found on the surface of the panel and it was very sticky to the surface. Some spots of water were also found on the panel results restricted in incoming solar radiation on panel surface. A thick layer on the 
system A was also found as the panel was not washed.

The use of surfactants seems feasible as the consumption of surfactants was very low as compared to increase in maximum power generation. By spending INR 1.274 in surfactants, we are able to generate $27.5 \%$ more power in 90 days.

\section{REFERENCES}

[1] (2019,july)ibef.[Online].https://www.ibef.org/industry/rene wable-energy-presentation

[2] (2019, july) mnreindia.[Online]. https://mnre.gov.in/ physical- progress-achievements

[3] Mazumder M.K., Horenstein M.N., Joglekar N.R., Sayyah A., Stark J.W., Bernard A.A.R., Garner S.M., Yellowhair J.E., Lin H.Y., Eriksen R.S., Griffin A.C., Gao Y., Centra R.L., Lloyd A.H., "Improvement of an electrostatic cleaning system for removal of dust from solar panels," Elsevier, 2017.

[4] Patil P.A., Bagi J.S., Wagh M.M., Patil G.S., "design optimization, automation and testing analysis of dust cleaning mechanism for solar photovoltaic power plant," 2019.

[5] Kawamoto H., "Electrostatic cleaning equipment for dust removal from soiled solar panels," Elsevier, 2019.

[6] Kawamoto H., Guo B., "Improvement of an electrostatic cleaning system for removal of dust from solar panels," Elsevier, 2018.

[7] Mozumder M.S., Mourad A.-H.I., Pervez H., Surkatti R.," Recent developments in multifunctional coatings for solar panel applications: A review,2019.

[8] Walz K.A., Shoemaker J.B., Scholes A.J., Jiang H., Silva J.M.S., Sanfilippo J., Zeltner W.A., Anderson M.A., " Experimental field trial of self-cleaning solar photovoltaic panels," 2018.

[9] K.A. Moharram, "Influence of cleaning using water and surfactants on the performance," Elsevier, 2013.

[10] (2019, july) MNIT [Online]. https://www.moniee.com/mnit 